\title{
Nonlinear analysis of RR and QT variability for cosmonauts data
}

\author{
A.V.Martynenko ${ }^{1}$, N.Yabluchansky ${ }^{1}$, A.Yabluchansky ${ }^{1}$, B.Verheyden $^{2}$, F.Beckers $^{2}$ and A.E.Aubert ${ }^{2}$ \\ ${ }^{1}$ School of Medicine, National University, Kharkov, Ukraine \\ ${ }^{2}$ Dept. of Cardiology, University Hospital Gasthuisberg, K.U. Leuven, Leuven, Belgium
}

\begin{abstract}
Nonlinear analysis of cardiovascular oscillations gives important information about their autonomic regulations. New nonlinear tools like Mindexes gives possibility to analyze the most pure dynamic process that are observed by $R R$ and QT intervals variability. Time series classification that is based on $M-$ indexes allows using stationary methods of oscillation variability like Fourier analysis for sure.
\end{abstract}

Keywords - RR variability, QT variability, nonlinear variability analysis, $M$-indexes

\section{INTRODUCTION}

Changing gravity conditions especially space flight has an important impact on the cardiovascular system [1]. Variability of cardiovascular oscillations has become a universal tool to study the neural control of the heart i.e. the delicate interaction between sympathetic and vagal influences on heart rate [2]. A variety of linear, non-linear, periodical and non-periodical oscillation patterns are presented in heart rate fluctuations. Linear methods of variability analysis are developed for study regular oscillatory patterns and cannot define nonlinear processes or fluctuations. The nonlinear methods represent promising tools for variability assessment but standards are lacking and the full scopes of these methods have not been fully assessed [2]. This is especially concerning new methods that incorporate for analysis of both nonlinear and linear behaviour in variability of cardiovascular oscillations time series.

The present study shows comparative different linear and nonlinear methods for RR and QT variability of cosmonauts before and after space flight.

\section{MATERIALS AND METHODS}

Subjects: 4 cosmonauts; 20 days before space flight (4 records) and on the $1^{\text {st }}$ ( 3 records), $4^{\text {th }}$ ( 3 records) and $5^{\text {th }}$ ( 1 record $), 7^{\text {th }}$ ( 1 record $)$ and $9^{\text {th }}(3$ records $)$ days after space flight.

Signal acquisition: 2 minutes of 2 channels high resolution $(1 \mathrm{kHz})$ digital ECG recording was made by Prof. A.Aubert group. Automatic RR and QT detection and verification was made by Prof. A.Martynenko group.

Spectral RR and $Q T$ variability analysis: RR and QT variability parameters in time and frequency domain were calculated with the following standards of measurement by the Task Force of ESC and NASPE [3]. In the time domain: mean and standard deviation for RR and QT intervals. In the frequency domain: total power (TP), very low frequency power (VLF), low frequency power (LF), high frequency power (HF) and $\mathrm{LF}$ to $\mathrm{HF}$ ratio (LF/HF).

Nonlinear RR and $Q T$ variability analysis: $\mathrm{RR}$ and $\mathrm{QT}$ nonlinear measurement parameters include both well known nonlinear methods Approximate Entropy (ApEn) [2, 4], Correlation Dimension (D2) [4], Hurst index (H-index) [5] and novel developed M-index [6].

We understand:

$A p E n$ as a statistic quantifying regularity and complexity of a stationary signal. ApEn quantifies the predictability of fluctuations in the time series [4];

D2 based on the Grassberger-Procaccia algorithm and measures geometrical properties of nonlinear dynamic process that represent the observed time series [6]. Higher values of D2 indicate more complexity in system [4];

$H$-index is the absence of pattern measure of time series that classify data as persistent, ergodic or Gaussian and define probability space for observed time series [5];

M-index is a complex measure of nonlinearity and nonstationarity of time series in both time symmetric $\left(\mathrm{M}_{0}\right)$ and time asymmetric $\left(\mathrm{M}_{1}\right)$ phase space. $\mathrm{M}$-index calculated in comparison with exponential time process and defined as local Lyapunov exponents [6].

We suggest time series classification that is based on Mindexes and our investigation of processes with different probability distributions [6]:

$|\mathrm{M}|<0.03$ - linear, stationary;

$0.3>|\mathrm{M}|>0.03$ - weakly nonlinear, quasi stationary;

$3>|\mathrm{M}|>0.3$ - nonlinear, transient;

$|\mathrm{M}|>3$ - strongly nonlinear, transient.

It is strong evidence that frequency domain variability analysis should be executed only for records or part of records with $|\mathrm{M}|<0.3$ [6].

\section{RESULTS}

Physiologically we understand QT intervals as a kernel part for correspondent RR intervals [7]. It is proved that QT dynamic changes are much less in comparison with RR but still correlate well with heart rate.

The purpose of current investigation is to find out the best measure that represents itself kernel dynamic process observed by QT and RR intervals variability. It means that such measure should be optimally correlated when calculated separately for QT and RR intervals because there is less possible stochastic part of both observations. 


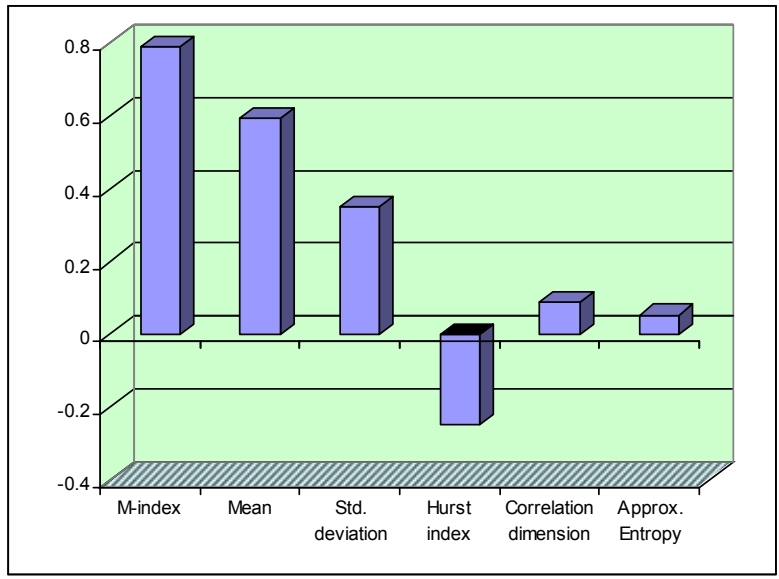

Fig. 1 Correlation between RR and QT in time domain and nonlinear analyzing measures

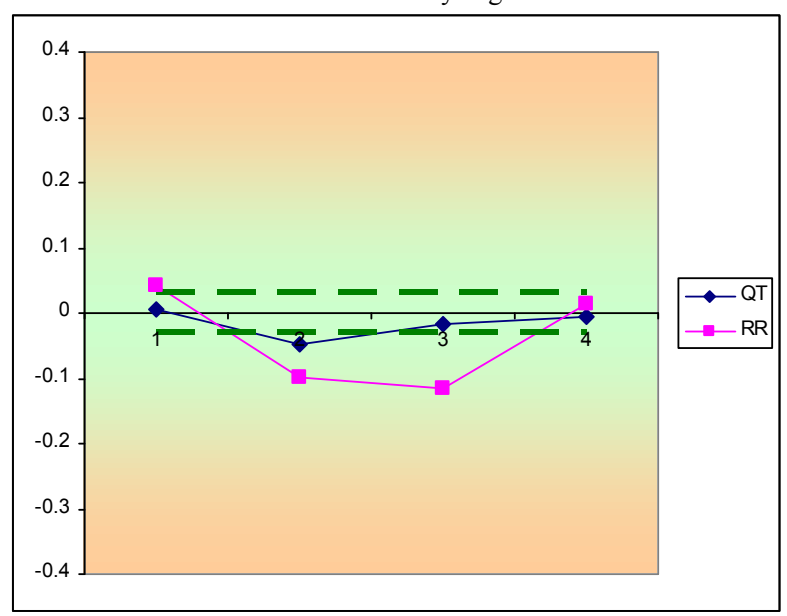

Fig. 3 M-indexes for cosmonaut A data

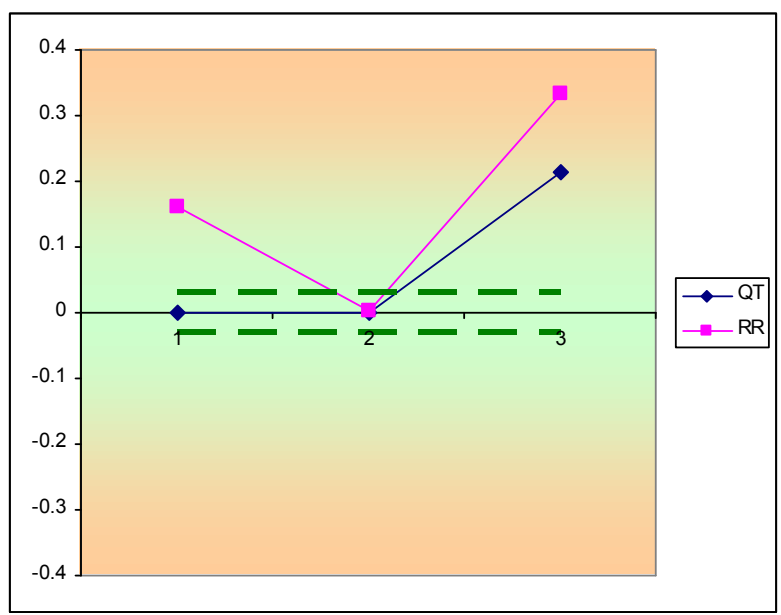

Fig. $5 \mathrm{M}$-indexes for cosmonaut $\mathrm{C}$ data

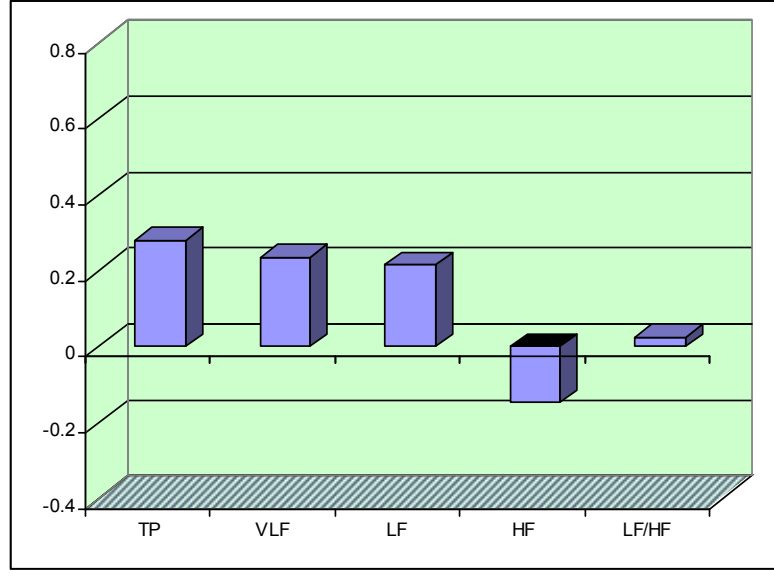

2 Correlation between RR and QT in frequency domain measures Fig.

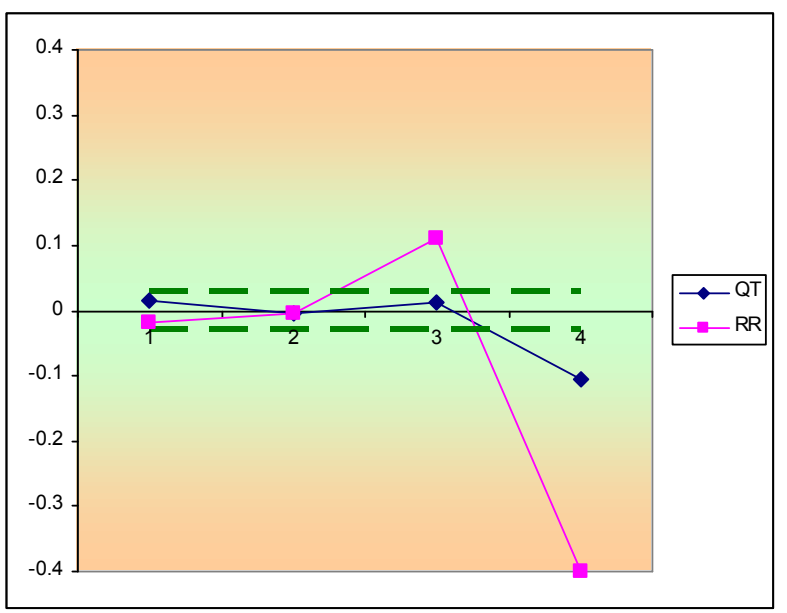

Fig. 4 M-indexes for cosmonaut B data

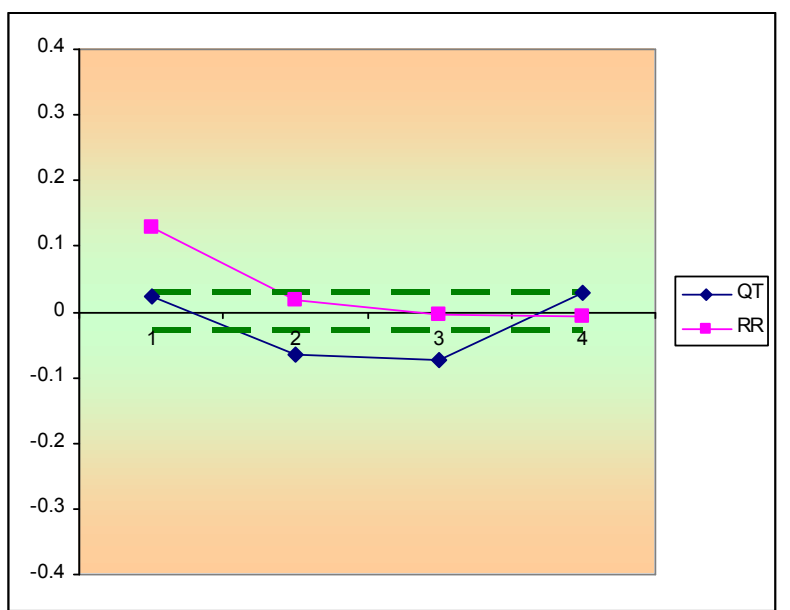

Fig. $6 \mathrm{M}$-indexes for cosmonaut $\mathrm{D}$ data 
The results of calculations Pearson correlation coefficient for RR and QT data analysis are shown in Figure 1 for time domain and nonlinear analysis and in Figure 2 for frequency domain analysis. The highest correlation coefficient $r=0.79$ shows M-index; after that Mean for intervals $r=0.59$. Frequency domain parameters show weak correlation because as we expected correlation for Standard Deviation is quite small $r=0.35$. We can consider measurement of ApEn, D2 and LF/HF as uncorrelated. Hurst index and HF show weak inverse correlation.

As far as M-index is the best representation of dynamic regulatory process that we observed by RR and QT intervals variability we learned cosmonauts' data evaluation before and after space flight relying on M-index. The Figures 3-6 show M-index for every cosmonaut.

First of all, we should ascertain that in all cases nonlinearity of QT data (mean for absolute value 0.025 ) less than RR (mean for absolute value 0.168). Secondly - space flight disturbances push $\mathrm{M}$-index in downside that means shrinkage of variability in the phase space. The last but not the least we notify two groups of index behaviour: return to stability zone after disturbances - cosmonauts A and D; uncompensated motion for the period of observation cosmonauts $\mathrm{B}$ and $\mathrm{C}$.

\section{DISCUSSION}

The short cardiovascular variability records especially heart rate variability are non stationary and poor for analysis by standard spectral methods. The better result can be obtained by analysis of QT intervals variability. We should use new methods like M-index measure that incorporate both linear and nonlinear analysis of dynamical process for study total data of variability cardiovascular oscillations. The stability of data should be added for further nonlinear data investigation.

\section{CONCLUSIONS}

The present study introduces M-index as one of the best measure of kernel nonlinear dynamic process that we can observe by RR and QT intervals variability. We have shown that nonlinearity of QT less than RR; space flight disturbances shrunk variability in phase space; nonlinear variability has individual behaviour that can be consolidated in common behaviour groups. M-index as measure of nonlinearity and nonstationarity is good for testing of biosignal records for the purpose of it further analysis by different stationary methods.
1. Aubert A., Beckers F., Verheyden B. Cardiovascular function and basics of physiology in microgravity. Acta Cardiol, 2005; 60: 129151.

2. Beckers F., Ramaekers D., Aubert A. Approximate Entropy of Heart Rate Variability: Validation of Methods and Application in Heart Failure. Cardiovasc. Eng., 2001; 1(4): 177-182.

3. Heart rate variability: standards of measurement, physiological interpretation and clinical use. Task force of the European Society of Cardiology and the North America Society of Pacing and Electrophysiology. Circulation, 1996; 93/5: 1043-1065.

4. Beckers F. Linear and Nonlinear Dynamics of Cardiovascular Variability - Validation \& Clinical Applications. Leuven Univ. Press, 2002.

5. Mandelbrot B. Statistical Methodology for Non-Periodic Cycles: from the Covariance to R/S Analysis. Annals of Economic Social Measurement, 1972; 1.

6. Yabluchansky N., Martynenko A., Isaeva A., Zinkovich I., Hripachenko I. Investigation of cardiovascular regulatory processes. Donetsk Press, 2005.

7. Coumel P., Maison-Blanche P. Heart rate variability and QT intervals: their relationships with the cardiac frequency. In.: Aubert A., Ector H., Stroobandt R. Cardiac Pacing and Electrophysiology, Kluwer Academic Publ., 1994: 63-72.

Corresponding author:

Alexander Martynenko

National University

4 Svoboda Sq.

Kharkov

Ukraine

may@univer.kharkov.ua

\section{REFERENCES}

